\title{
Optimization of DNA Sensor Model Based Nanostructured Graphene Using Particle Swarm Optimization Technique
}

\author{
Hediyeh Karimi, ${ }^{1,2}$ Rubiyah Yusof, ${ }^{1,2}$ Rasoul Rahmani, ${ }^{1}$ and Mohammad Taghi Ahmadi ${ }^{3,4}$ \\ ${ }^{1}$ Centre for Artificial Intelligence and Robotics, Universiti Teknologi Malaysia, 54100 Kuala Lumpur, Malaysia \\ ${ }^{2}$ Malaysia-Japan International Institute of Technology (MJITT), Universiti Teknologi Malaysia, 54100, Malaysia \\ ${ }^{3}$ Computational Nanoelectronic Research Group, Faculty of Electrical Engineering, Universiti Teknologi Malaysia, \\ 81310 Johor, Malaysia \\ ${ }^{4}$ Physics Department, Nanotechnology Research Center Nanoelectronic Group, Urmia University, Urmia 57147, Iran
}

Correspondence should be addressed to Rubiyah Yusof; rubiyah@ic.utm.my

Received 14 July 2013; Revised 5 October 2013; Accepted 6 November 2013

Academic Editor: Munawar A. Riyadi

Copyright (C) 2013 Hediyeh Karimi et al. This is an open access article distributed under the Creative Commons Attribution License, which permits unrestricted use, distribution, and reproduction in any medium, provided the original work is properly cited.

It has been predicted that the nanomaterials of graphene will be among the candidate materials for postsilicon electronics due to their astonishing properties such as high carrier mobility, thermal conductivity, and biocompatibility. Graphene is a semimetal zero gap nanomaterial with demonstrated ability to be employed as an excellent candidate for DNA sensing. Graphene-based DNA sensors have been used to detect the DNA adsorption to examine a DNA concentration in an analyte solution. In particular, there is an essential need for developing the cost-effective DNA sensors holding the fact that it is suitable for the diagnosis of genetic or pathogenic diseases. In this paper, particle swarm optimization technique is employed to optimize the analytical model of a graphene-based DNA sensor which is used for electrical detection of DNA molecules. The results are reported for 5 different concentrations, covering a range from $0.01 \mathrm{nM}$ to $500 \mathrm{nM}$. The comparison of the optimized model with the experimental data shows an accuracy of more than $95 \%$ which verifies that the optimized model is reliable for being used in any application of the graphene-based DNA sensor.

\section{Introduction}

In the recent decade, DNA biosensors have been widely considered as a promising means of diagnostic prediction in genetic research such as cancer or hereditary disease due to their rapid and sensitive response, as well as the simple and convenient operation $[1,2]$. The interface between nanomaterials and biosystems is emerging as one of the most various and dynamic areas of intense research [3]. In order to overcome the limit of planar semiconductor devices regarding sensitivity and potential for integration $[4,5]$, these nanomaterials are commonly proposed in sensor platform. Nanoscale channel confinement of planar FETs makes them extremely sensitive to electric perturbations, due to their one-dimensional structures. Therefore, obtaining a high sensitivity does not provide a worthy and reliable sensor. The implementation of nanomaterials of graphene in graphene field effect transistor (GFETs) constitutes an important step toward low-cost, easy handle, and highly sensitive molecular diagnostics $[6,7]$. On the other hand, graphene with a two-dimensional thick monoatomic block of a carbon allotrope offers a large detection area (as seen in Figure 1) for DNA sensing compared with other known nanomaterials [8-11].

The research to date tends to focus on the impact of graphene-based materials in electronic and optoelectronic devices along with their potential applications [6].

\section{Materials and Methods}

2.1. Graphene-Based DNA Sensor Structure. Over the past few decades, a lot of information has become available on fieldeffect devices for DNA detection which is reported in the literature [12-21]. DNA sensors usually presented in diverse sensor configurations such as electrolyte-silicon (ES) structures, depletion- and enhancement-mode FETs, floating-gate 


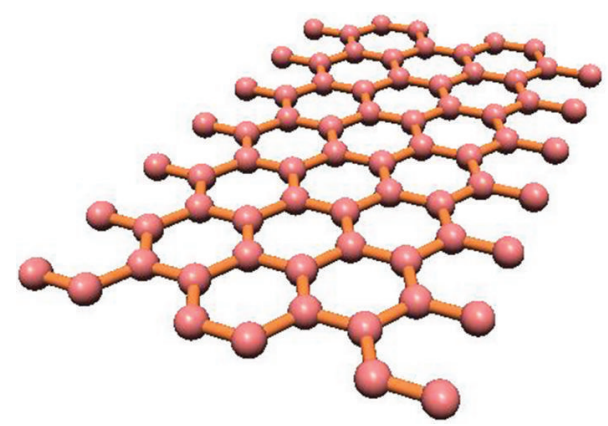

FIGURE 1: Monolayer graphene structure with one atom thickness.

FET, and FET devices with or without a reference electrode. $\mathrm{SiO}_{2}$, silanized $\mathrm{SiO}_{2}, \mathrm{SiO}_{2}-\mathrm{Si}_{3} \mathrm{~N}_{4}, \mathrm{SiO}$-poly-L-lysine were employed as various gate-insulator materials with different thicknesses (from 2 to $100 \mathrm{~nm}$ ) in various DNA immobilization methods (such as adsorption, covalent attachment, biotin-vidin complexation, and linker molecules) [22]. As reported in [16], the densities of the immobilized DNA varies from $2.4 \times 10^{8}$ to $5 \times 10^{13}$ molecules $/ \mathrm{cm}^{2}$ and hybridization buffer solutions with different electrolyte concentrations (from $10 \mathrm{nM}$ to $1 \mathrm{nM}$ ) were examined to obtain the higher adsorption. In addition, the mostly reported flat-band or threshold voltage shift induced upon the DNA hybridization reach from several $\mathrm{mV}$ up to around $1.9 \mathrm{~V}$. The focus of this theoretical study will be on developing the DNA-sensorsbased graphene nanomaterials which have emerged as one of the most popular devices holding great promise to provide a straightforward platform of patience diagnosis [23, 24].

As shown in Figure 2, the schematic of the DNA sensor consists of a 300-nm $\mathrm{SiO}_{2}$ layer as a back-gate dielectric and a doped silicon substrate as the back gate which was proposed for modeling of DNA sensor in our previous studies [9, 25]. Based on that, graphene layer acts as a conducting channel connected to the source and drain electrodes which are made from $\mathrm{Au}$. An $\mathrm{Ag} / \mathrm{AgCl}$ reference electrode commonly acts as a gate for measuring the electrical characteristics in a liquid-gated or electrochemically gated configuration that controls the current along the graphene sheet between the two electrodes [26-28]. Considering that the gate leakage effect is not considered in the current study, the DNA sensors were exposed to a phosphate buffer solution (PBS) containing the DNA molecules. In order to understand whether DNA molecules are adsorbed on the graphene surface or not, source-drain conductance were measured before and after exposing the DNA sensor to the different concentration of DNA molecules [3].

It is noteworthy to explain the DNA adsorption effect on nanomaterials of graphene surface, as well as the proposed model. The FET devices are very practical for measuring the surface charge and therefore the charge changes because a DNA adsorption event can be measured with a high accuracy. It has been demonstrated that the interaction between DNA molecules and graphene layer causes a significant change in the conductance of the graphene-based DNA sensor through the nonelectrostatic gating mechanism
$[29,30]$. In Figure 3, the effect of DNA concentration on the gate voltage at nanomolar concentrations is explored. It is generally suggested that the electronic-doping ( $n$-doping) effect by the adsorbed charge species has a significant impact on conductance change of graphene-based transistors [31]. Moreover, the conductivity of the graphene-based DNA sensor is influenced by the increased number of carriers in the channel. As the number of DNA molecules increased from 0.01 to $500 \mathrm{nM}$, due to the negative charges of DNA molecules, it could be expected that the gate voltage would shift leftwards to the lower amounts $[32,33]$. Based on the detection mechanism, we recently proposed an analytical model for detection of DNA molecules in which the DNA concentration was modeled by a gate voltage [9].

Although there are lots of works presented on the experimental progress, the detection mechanism is not understood quantitatively. On the other hand, modeling and simulation using partial differential equations (PDE) play a critical role in determining the current-voltage characteristics, sensitivity, and the behaviour of the sensing devices exposed to DNA molecules. Our proposed model is capable of performing the electrical detection of DNA molecules by modeling the conductance of the graphene sheets. The conductance of the large channel in graphene materials is obtained in the ohmic scaling law using Landauer formula [34].

Consider

$$
G=\frac{2 q^{2}}{h} \int_{-\infty}^{+\infty} d E M(E) T(E)\left(-\frac{d f}{d E}\right),
$$

where $q$ is the electron charge, $h$ denotes Planck's constant, $E$ represents the energy band structure, $T(E)$ is the transmission probability, $M(E)$ is the number of modes, and $f$ denotes the Fermi-Dirac distribution function $[34,35]$. In other words, $T(E)$ is the average probability of electron transmission in the channel from one electrode to another. Since the channel is assumed ballistic, the probability is considered equal to one $(T(E)=1)$ [36-38]. In order to highlight the effect of DNA adsorption on graphene, DNA concentration as a function of gate voltage is assumed and sensing factor is defined. High carrier mobility reported from experiments in the graphene leads to assume a completely ballistic carrier transportation in the graphene [39]. In the nonsaturation region, the DNA concentration model is employed as a function of gate voltage and the ideal current-voltage relation for the n-channel FET from [34] is modified as

$$
\begin{aligned}
I_{d}= & \frac{3 q^{2}\left(3 \pi a^{3} t^{3} k_{B T}\right)^{1 / 2}}{h L} \\
& \times\left[\mathscr{I}_{-1 / 2}(\eta)+\mathscr{I}_{-1 / 2}(-\eta)\right] \\
& \times\left(\frac{\propto}{F} V_{g s(\text { withoutDNA })}-V_{t}\right),
\end{aligned}
$$

where $a_{\mathrm{C}-\mathrm{C}}=1.42 \AA$ is carbon-carbon $(\mathrm{C}-\mathrm{C}$ ) bond length, $t=$ $2.7(\mathrm{eV})$ is the nearest neighbor $\mathrm{C}-\mathrm{C}$ tight binding overlap energy, $L$ is the length of conducting channel, $V_{t}$ refers to the threshold voltage, $\propto$ is DNA sensing factor, and $F$ is DNA concentration. Different concentrations of DNA molecules 


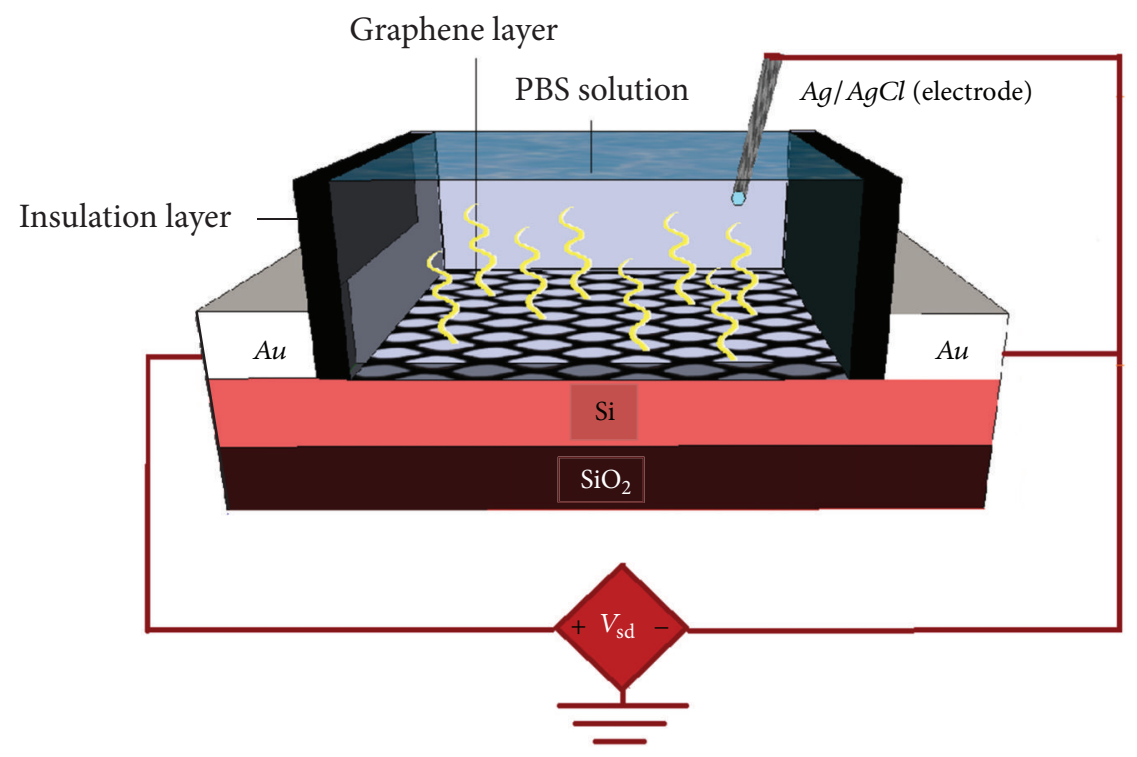

FIGURE 2: Schematics of DNA sensor measurement setup operated by liquid gating where a device is gated through an $\mathrm{Ag} / \mathrm{AgCl} \mathrm{reference}$ electrode inserted in the electrolyte.

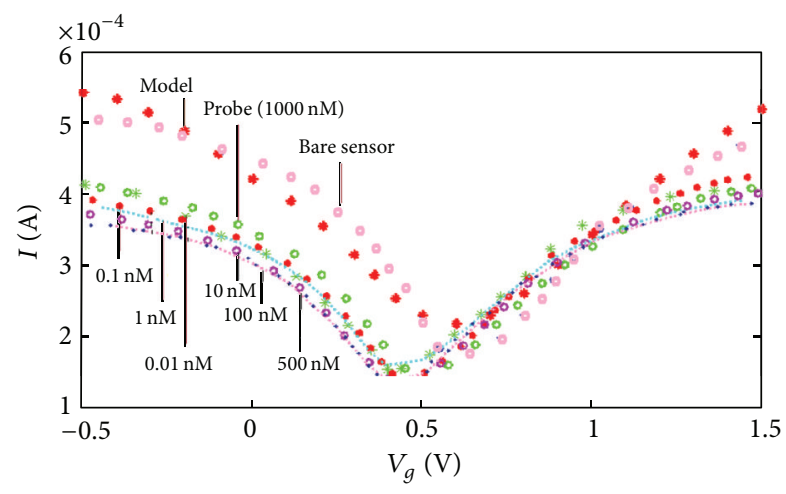

Figure 3: Transfer characteristics of graphene FET for different concentrations of DNA molecule.

were presented in the form of $F$ parameter. Thus, the DNA molecules adsorbed on graphene surface by iteration method are modeled as

$$
\propto=A F^{2}+B F+C
$$

From extracted data, $(A, B, C)$ parameters are calculated and obtained as $A=13, B=50$, and $C=4070$. Eventually, according to the proposed model of DNA sensor using nanostructured graphene layer, the current-voltage characteristic is modified as

$$
\begin{aligned}
I_{d}= & \frac{3 q^{2}\left(3 \pi a^{3} t^{3} k_{B T}\right)^{1 / 2}}{h L} \\
& \times\left[\mathscr{I}_{-1 / 2}(\eta)+\mathscr{I}_{-1 / 2}(-\eta)\right] \\
& \times\left(\frac{13 F^{2}+50 F+4070}{F} V_{g s(\text { withoutDNA) }}-V_{t}\right) .
\end{aligned}
$$

All theoretical $I_{d}-V_{g}$ characteristics of graphene FET for DNA concentration are plotted in [30] changing from 0.01 to $500 \mathrm{nM}$. It is stated that the sensor model with the proposed parameters represents the same trends as those reported as experimental data [3,9]. Since the values of the parameters $A, B$, and $C$ in (3) are calculated based on trial and error, there is necessity of a methodological approach for obtaining a viable and accurate model which is reliable for being used in different applications of the graphenebased DNA sensor. To this aim, a swarm-based evolutionary algorithm (EA) called particle swarm optimization (PSO) is used for optimizing the mathematical model shown in (3). In important applications of DNA sensors such as hybridization detection, the behaviour of $I_{d}-V_{g}$ characteristic around $V_{g \text { min }}$ is very important and determinant. Therefore, a proper and reliable model should be capable of providing the same features with experimental data around the mentioned area. The better the modelled curve fits the experimental result, the more the reliable model is achieved. In the current study, the optimized model using PSO provides a fitter $I_{d}-V_{g}$ characteristic compared with the previous mathematic model, especially around the $V_{g \text { min }}$ point which is very important for the main applications of the model.

The PSO technique is widely used in optimizing different sorts of problems including fine materials, medical science, control theory, and energy issues [40-43]. The important facts which make PSO popular among the researchers are its fastness, its ability to avoid being trapped in the local optima, and its capability of being employed in any type of optimization problem [44-48]. Swarm intelligence techniques have several advantages that make them useful for optimization applications. Some of these advantages are scalability, flexibility, robustness, and low production costs. Scalability means particles can be added to the swarm without making any change in the overall behaviour of the colony. In other words, 


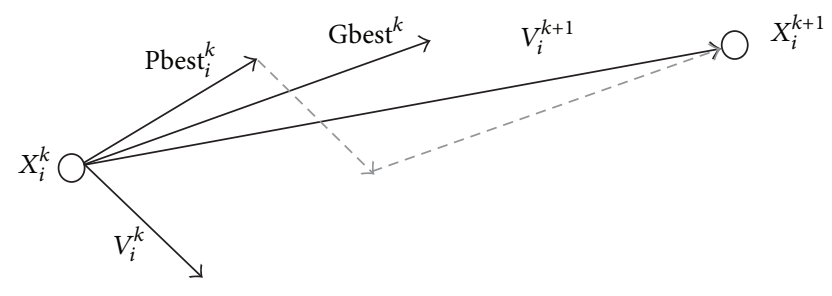

FIGURE 4: A simple diagram for movement of a sample particle in PSO.

individuals typically have a limited range of interaction with their neighborhood, so that the introduction of a new particle does not directly affect the whole swarm [49]. That helps to change the scale and size of the swarm easily, based on the size of the search space defined. Flexibility gives the ability to the swarm to be adapted to different problems under different circumstances. A well-designed swarm algorithm can exploit and explore an unknown territory, even encountering a huge gap in the search space. With regard to the robustness, if a few faulty individuals exist in the swarm, they may not affect the global optimum found by the swarm. The fourth main advantage is having low production costs. This is respective to the computational cost and memory size per unit of the individuals which gives the possibility of being used in small cheap components. The main advantage of the particle swarm optimization technique over other global optimization methods such as simulated annealing is having a large number of agents which makes the particle swarm technique resilient to the local optima points of the search space. In other words, it helps the algorithm in not being trapped in the local optima points.

2.2. Particle Swarm Optimization (PSO). The PSO algorithm is mainly based on the movement of a population of particles as a "Swarm." This movement is inspired from a school of fish or a bird flock [50-52]. The evaluation of the search space is being performed step by step. There are some parameters which need to be saved at each step, since they will be needed in calculating the parameters of the next step. Equations (4) and (5) show the velocity and location of $i$ th particle at $k$ th step.

Consider

$$
\begin{gathered}
V_{i}^{k+1}=W \times V_{i}^{k}+c_{1} \times r_{1}\left(\text { Gbest }_{i}^{k}-X_{i}^{k}\right) \\
+c_{2} \times r_{2}\left(\text { Pbest }_{i}^{k}-X_{i}^{k}\right), \\
X_{i}^{k+1}=X_{i}^{k}+V_{i}^{k},
\end{gathered}
$$

$i=1,2, \ldots$, nop (number of particles); $k=1,2, \ldots, k_{\max }$ (maximum iteration number), where, $K$ is the iteration number; $i$ is the particle number; $W$ refers to the inertia weight coefficient which is normally chosen between 0.5 and 1.2; $c 1$ and $c 2$ are acceleration constants; $r 1$ and $r 2$ are random values between 0 and $1 ; V_{i}^{k}$ is the velocity of particle $i$ at iteration $k$; and $X_{i}^{k}$ indicates the position of particle $i$ at iteration $k$.

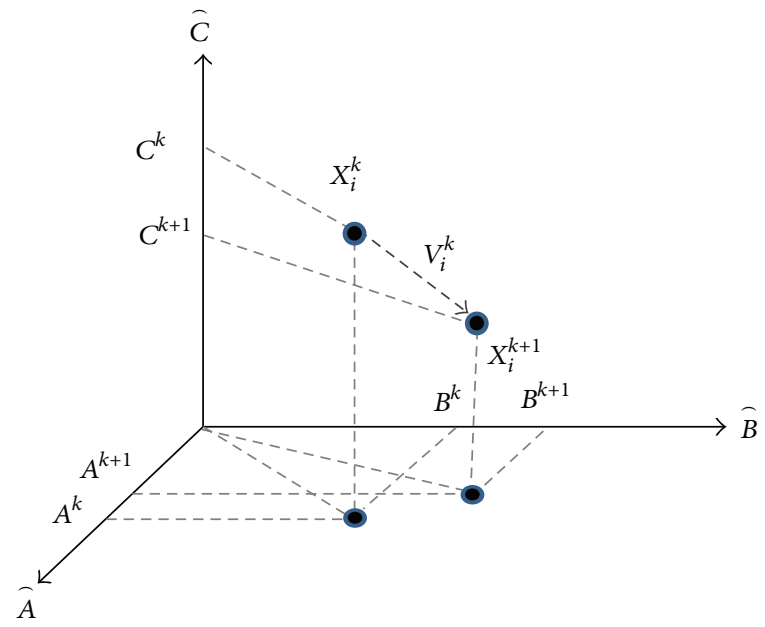

FIGURE 5: The search pace of the problem.

Besides the above parameters which should be calculated for each particle, there are some social parameters that lead the swarm to the region which contains the global optima. "Personal best" and "Global best" are two parameters which are being used in all the PSO versions. They are normally shown as "Pbest" and "Gbest," respectively. The Pbest is known as the best location that each particle experienced so far during the runtime, and the Gbest is the best global optimum found so far by the swarm. Figure 4 shows a simple diagram of movement of a typical particle.

\section{Implementing Particle Swarm Optimization in DNA Sensor}

3.1. Representation of the Search Space. Swarm is the particles which are moving and giving solutions for solving the problem. The particles move in the domain of the problem space and each of them represents a solution for the problem. The parameters to be optimized in the DNA sensor model are $A, B$, and $C$ coefficients as in (2). Figure 5 illustrates a three-dimensional search space of the problem. If $\widehat{A}, \widehat{B}$, and $\widehat{C}$ are the vectors which return $A, B$, and $C$, respectively, then particle $i$ flies in the search space to find the best possible values for the respective parameters. Vector $V_{i}$ is the movement velocity vector of the particle $i$ which is obtained from (4).

In the optimization process, each location represented by the particles is a possible solution. The search space consists of arrays and matrices. For example, $X[i][j]$ is a matrix of the location of the particles in the proposed algorithm, while $i$ indicates the particle and $j$ is the index of the optimizing parameter. In Figure 5, the dimension of the $X[i][j]$ matrix will be $200 \times 3$, if we consider the number of particles to be equal to 200 .

3.2. Fitness Function and Parameter Selection. To evaluate the solutions proposed by the particles, we need to define a fitness function. The fitness function has to be able to 


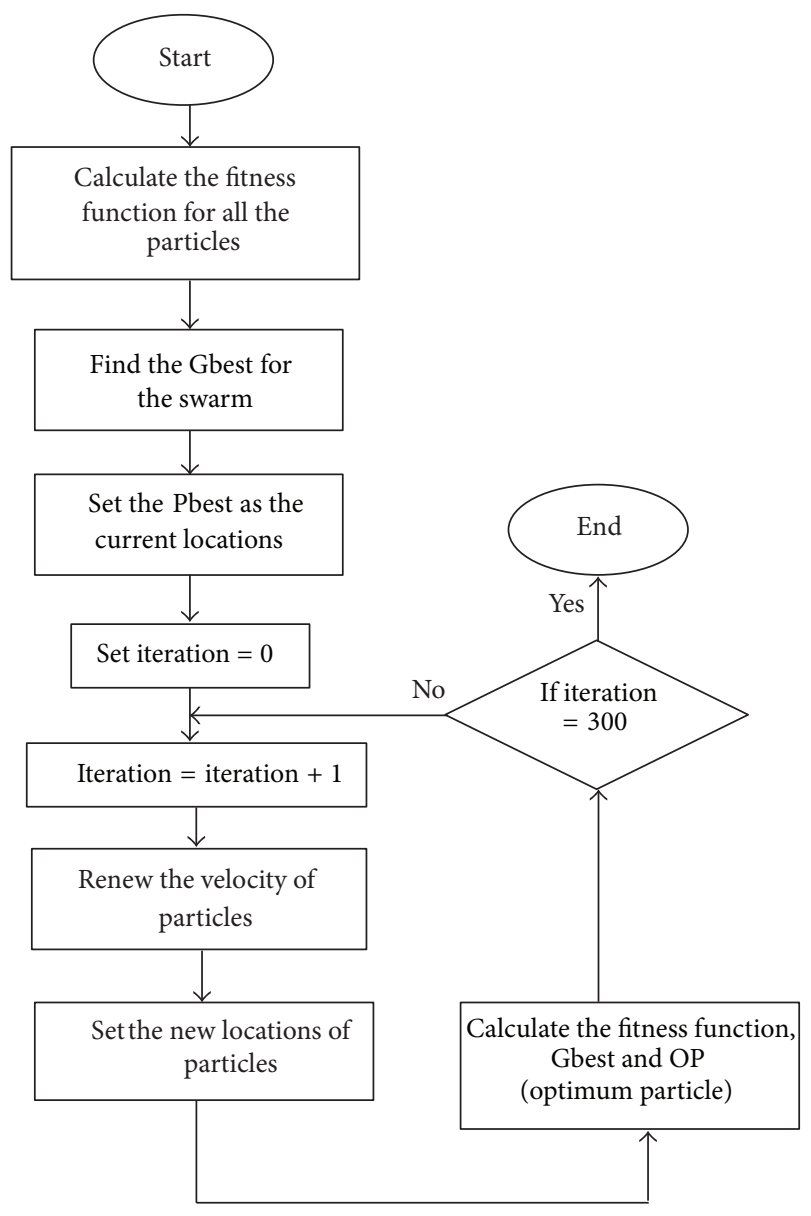

FIGURE 6: A flowchart of PSO-based algorithm for optimizing the DNA sensor.

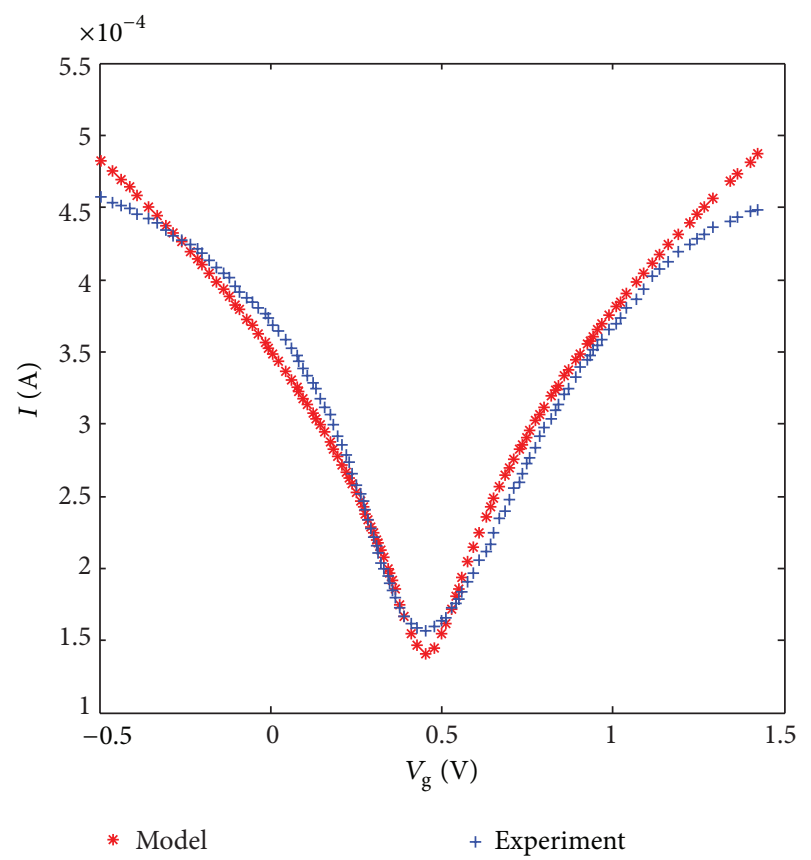

FIGURE 7: The experimental and optimized model waveforms for the bare DNA sensor.

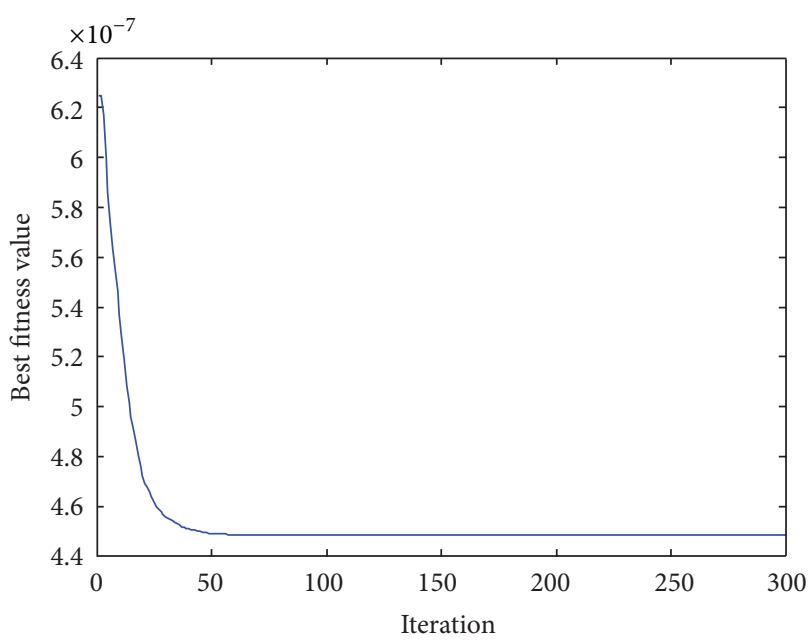

FIGURE 8: The convergence profile of the optimization process using PSO technique.

determine which solution is better and more efficient after considering all the solutions obtained by the particles at each iteration. Normally the fitness function is being set to have the lowest possible value at an optimum point. In the current study, we also need to have the lowest possible value of the error between the experimental waveform and the proposed model; hence, the fitness function is proposed as follows:

$$
\text { Fitness function }=\psi_{i}=\sum_{k=1}^{\max }\left(\widehat{I}_{i}(k)-I(k)\right)^{2} \text {, }
$$

where $\widehat{I}_{i}(k)$ represents the modelled current waveform for particle $i, I(k)$ is the experimental current of the DNA sensor, and $\psi_{i}$ is the fitness value of the $i$ th particle. Based on the fitness function chosen, the best solution will propose the most fitted curve for the current waveform which is desired for a suitable DNA sensor model. The parameters of the PSO algorithm are chosen based on the values tabulated in Table 1 , and the strategy of the optimization is shown in a flowchart as in Figure 6.

3.3. Optimization Results for DNA Sensor Model. After the optimization process, the best values obtained for the parameters $A, B$, and $C$ are tabulated in Table 2. These values are the best among the 20 runs of the optimization algorithm, and the lowest fitness value is the indicator of choosing the best parameters. The lowest obtained fitness value is also shown in the table which determines how good the modelled waveform fits the experimental curve.

The train for the optimizing parameters is performed using the experimental curve of the bare DNA sensor. Figure 7 illustrates the experimental waveform and the optimized model of the probe DNA sensor.

To show the convergence quality of the optimization process using PSO technique, the convergence profile of the best fitness value is shown in Figure 8 which is based on the fitness value versus iteration. The graph shows that the algorithm could converge to the optimized values after 


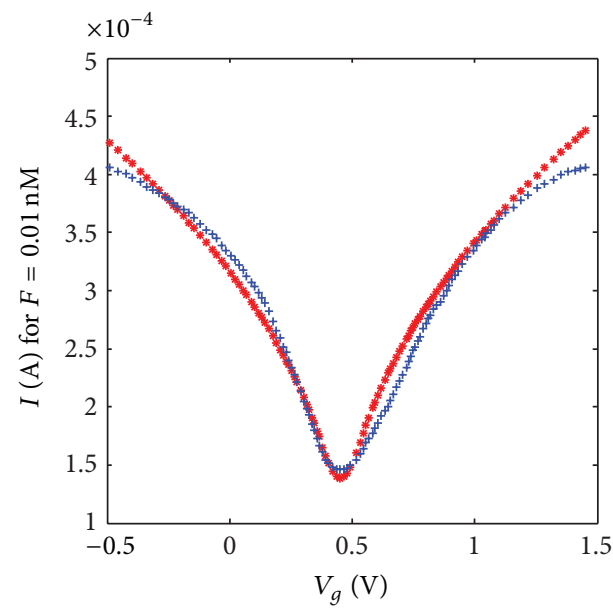

(a)

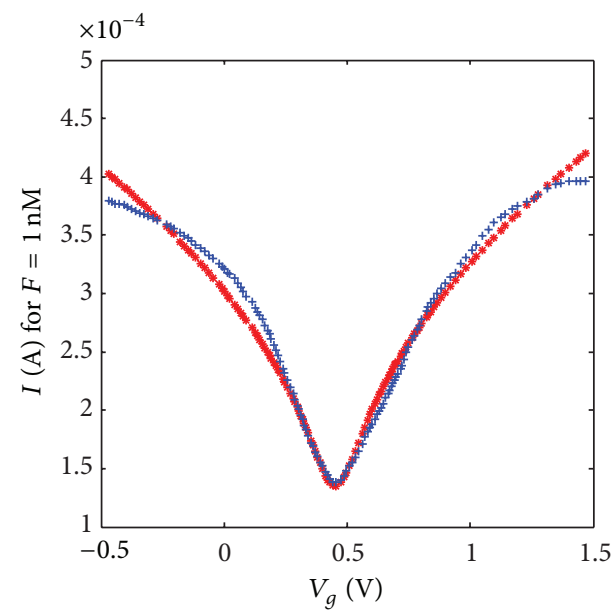

(c)

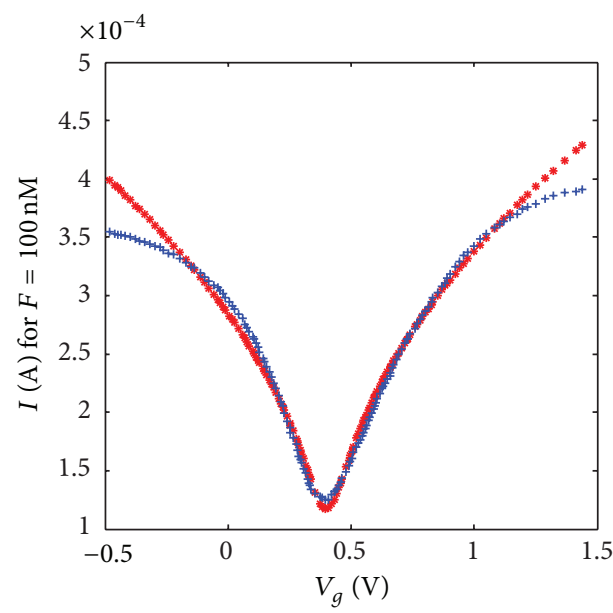

* Model

+ Experiment

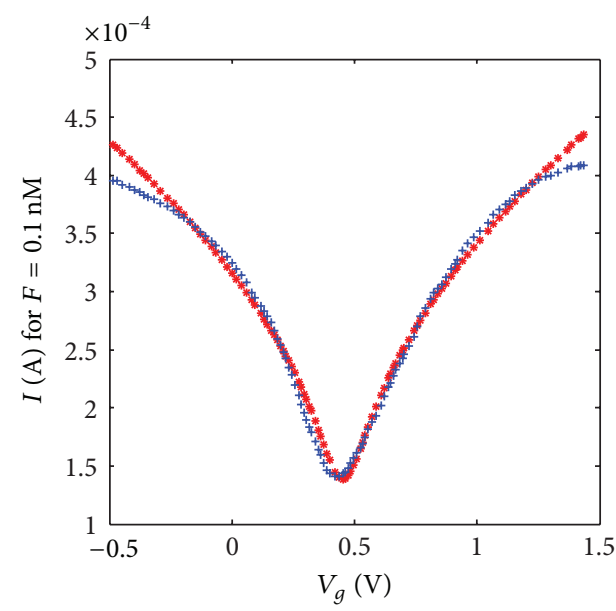

(b)

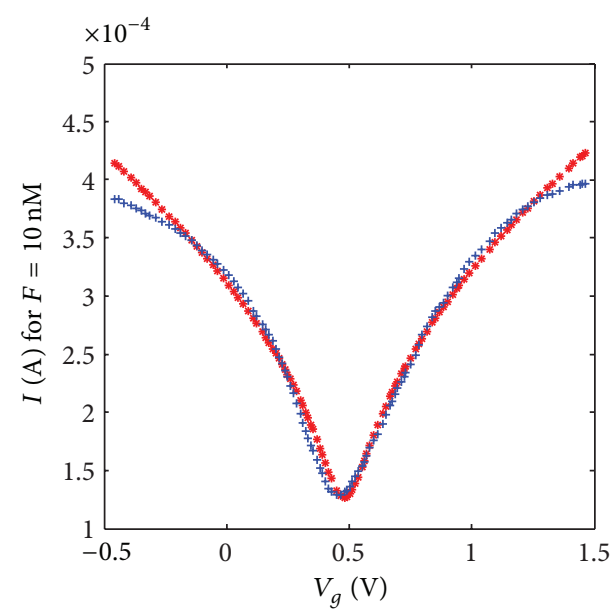

(d)

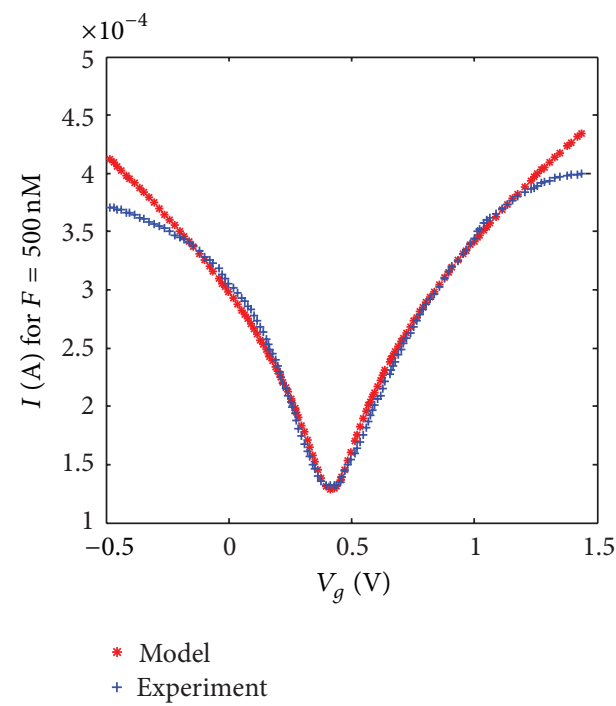

(f)

FIGURE 9: The optimized model and experimental waveforms for different concentrations of the DNA sensor: (a) $F=0.01 \mathrm{nM}$, (b) $F=0.1 \mathrm{nM}$, (c) $F=1 \mathrm{nM}$, (d) $F=10 \mathrm{nM}$, (e) $F=100 \mathrm{nM}$, and (f) $F=500 \mathrm{nM}$. 
TABLE 1: The parameters chosen for the PSO algorithm.

\begin{tabular}{lccccc}
\hline Number of particles (nop) & Number of dimensions (nod) & $c 1$ & $c 2$ & Maximum iteration & Inertia weight $(W)$ \\
\hline 200 & 3 & 2 & 2 & 300 & $0.4<W<1.2$ \\
\hline
\end{tabular}

TABLE 2: The best values of the optimizing parameters over the 20 runs.

\begin{tabular}{lccc}
\hline The best fitness value obtained & Optimized value for $A$ & Optimized value for $B$ & Optimized value for $C$ \\
\hline $4.484 e-07$ & $1.0865 e 10$ & $9.9662 e 9$ & $-5.921 e 3$ \\
\hline
\end{tabular}

TABLE 3: The MAPE value with respect to the different concentrations $(F)$.

\begin{tabular}{lcc}
\hline $\begin{array}{l}\text { Concentration } F \\
(\mathrm{nM})\end{array}$ & $\begin{array}{c}\text { MAPE value } \\
(\%)\end{array}$ & $\begin{array}{c}\text { Accuracy based on MAPE } \\
(\%)\end{array}$ \\
\hline$F=0.01$ & 4.58 & 95.42 \\
$F=0.1$ & 3.52 & 96.48 \\
$F=1$ & 3.33 & 96.67 \\
$F=10$ & 3.39 & 96.61 \\
$F=100$ & 3.52 & 96.48 \\
$F=500$ & 3.94 & 96.06 \\
\hline
\end{tabular}

around 60 iterations which show a good convergence speed of the proposed method.

Using the values obtained for $A, B$, and $C$ parameters, different concentrations of the DNA sensor are modelled and compared with the experimental waveforms with the same concentrations. To evaluate the quality of the obtained waveforms with respect to the experimental results, mean absolute percentage error (MAPE) index is used as an error evaluation parameter as follows:

$$
\operatorname{MAPE}=\frac{1}{n} \sum_{k=1}^{n}\left|\frac{\hat{I}(k)-I(k)}{I(k)}\right|
$$

The results based on the MAPE index for different concentrations of the DNA sensor are shown in Table 3. The accuracy based on the MAPE value can be obtained as the result of deducting MAPE from 100 percent. Based on the results in the table, the accuracy of the optimized model is more than $95 \%$ for all the concentrations which is an acceptable range of accuracy.

Also, the optimized waveforms for different concentrations are seen in Figure 9, where for each concentration the optimized model and experimental waveforms are plotted. It needs to be mentioned that the middle part of the graph is very important, since the voltage used in the experiment is normally between 0 and 1 volts. The optimized model fits even better around the mentioned voltage range.

It needs to be mentioned that the $V_{g \text { min }}$ (minimum gate voltage) is a very sensitive indicator for detection of DNA molecules and the optimized model shows a better performance around the ( 0 and 1 volt) voltage range.

\section{Conclusion}

Due to the unique characteristics of the graphene such as special $\mathrm{C}-\mathrm{C}$ bonding, $1 \mathrm{D}$ structure, large surface-tovolume ratio, high conductivity, and biocompatibility, it has remarkable potential for biosensing material applications. The graphene-based DNA sensor has a similar structure to traditional metal-oxide-semiconductor FET (MOSFET) in which the gate is exposed to the electrolyte solution containing DNA molecules. In these sensors, DNA adsorption occurring on a graphene surface changes the $I-V$ characteristics of the transistors due to the intrinsic negative charge of DNA. In this research, DNA sensing factor which was presented in our previous work is optimized using particle swarm optimization (PSO) technique. The optimization process is performed in order to find the optimized values of fitting parameters in a current-voltage characteristic of DNA sensor model to predict graphene doping behavior in GFET devices. Based on the results obtained, an accuracy of more than $95 \%$ is obtained for the optimized model compared with the available experimental data. It can be concluded that the optimized grapheme-based DNA sensor model with the accuracy obtained is now suitable and reliable for being employed in different applications such as diagnosis of genetic and pathogenic diseases.

\section{Acknowledgments}

The authors would like to acknowledge the financial support from the Research University Grant of the Ministry of Higher Education of Malaysia (MOHE) under Project Grant: FRGS4F149. Also, thanks to the Research Management Center (RMC) of Universiti Teknologi Malaysia (UTM) for providing an excellent research environment in which to complete this work.

\section{References}

[1] J. L. Su, B.-S. Youn, W. P. Ji, J. H. Niazi, S. K. Yeon, and B. G. Man, "ssDNA aptamer-based surface plasmon resonance biosensor for the detection of retinol binding protein 4 for the early diagnosis of type 2 diabetes," Analytical Chemistry, vol. 80, no. 8, pp. 2867-2873, 2008.

[2] A.-L. Liu, G.-X. Zhong, J.-Y. Chen et al., "A sandwich-type DNA biosensor based on electrochemical co-reduction synthesis of graphene-three dimensional nanostructure gold nanocomposite films," Analytica Chimica Acta, vol. 767, pp. 50-58, 2013.

[3] X. Dong, Y. Shi, W. Huang, P. Chen, and L.-J. Li, "Electrical detection of DNA hybridization with single-base specificity 
using transistors based on CVD-grown graphene sheets," Advanced Materials, vol. 22, no. 14, pp. 1649-1653, 2010.

[4] Y. Cui, Q. Wei, H. Park, and C. M. Lieber, "Nanowire nanosensors for highly sensitive and selective detection of biological and chemical species," Science, vol. 293, no. 5533, pp. 1289-1292, 2001.

[5] P. R. Nair and M. A. Alam, "Performance limits of nanobiosensors," Applied Physics Letters, vol. 88, no. 23, Article ID 233120, 2006.

[6] V. Singh, D. Joung, L. Zhai, S. Das, S. I. Khondaker, and S. Seal, "Graphene based materials: past, present and future," Progress in Materials Science, vol. 56, no. 8, pp. 1178-1271, 2011.

[7] Y. Shao, J. Wang, H. Wu, J. Liu, I. A. Aksay, and Y. Lin, "Graphene based electrochemical sensors and biosensors: a review," Electroanalysis, vol. 22, no. 10, pp. 1027-1036, 2010.

[8] N. Mohanty and V. Berry, "Graphene-based single-bacterium resolution biodevice and DNA transistor: interfacing graphene derivatives with nanoscale and microscale biocomponents," Nano Letters, vol. 8, no. 12, pp. 4469-4476, 2008.

[9] X. Dong, X. Zhao, L. Wang, and W. Huang, "Synthesis and application of graphene nanoribbons," Current Physical Chemistry, vol. 3, no. 3, pp. 291-301, 2013.

[10] F. Chen, Q. Qing, J. Xia, and N. Tao, "Graphene field-effect transistors: electrochemical gating, interfacial capacitance, and biosensing applications," Chemistry, vol. 5, no. 10, pp. 2144-2153, 2010.

[11] M. Zheng, A. Jagota, E. D. Semke et al., "DNA-assisted dispersion and separation of carbon nanotubes," Nature Materials, vol. 2, no. 5, pp. 338-342, 2003.

[12] E. Souteyrand, J. P. Cloarec, J. R. Martin et al., "Direct detection of the hybridization of synthetic homo-oligomer DNA sequences by field effect," Journal of Physical Chemistry B, vol. 101, no. 15, pp. 2980-2985, 1997.

[13] J. Fritz, E. B. Cooper, S. Gaudet, P. K. Sorger, and S. R. Manalis, "Electronic detection of DNA by its intrinsic molecular charge," Proceedings of the National Academy of Sciences of the United States of America, vol. 99, no. 22, pp. 14142-14146, 2002.

[14] F. Wei, B. Sun, Y. Guo, and X. S. Zhao, "Monitoring DNA hybridization on alkyl modified silicon surface through capacitance measurement," Biosensors and Bioelectronics, vol. 18, no. 9, pp. 1157-1163, 2003.

[15] M. H. Abouzar, A. Poghossian, A. G. Cherstvy, A. M. Pedraza, S. Ingebrandt, and M. J. Schoening, "Label-free electrical detection of DNA by means of field-effect nanoplate capacitors: experiments and modeling," Physica Status Solidi A, vol. 209, no. 5, pp. 925-934, 2012.

[16] D.-S. Kim, Y.-T. Jeong, H.-J. Park et al., "An FET-type charge sensor for highly sensitive detection of DNA sequence," Biosensors and Bioelectronics, vol. 20, no. 1, pp. 69-74, 2004.

[17] D.-S. Kim, H.-J. Park, H.-M. Jung et al., "Field effect transistorbased bimolecular sensor employing a Pt reference electrode for the detection of deoxyribonucleic acid sequence," Japanese Journal of Applied Physics, vol. 43, no. 6, pp. 3855-3859, 2004.

[18] F. Uslu, S. Ingebrandt, D. Mayer, S. Böcker-Meffert, M. Odenthal, and A. Offenhäusser, "Labelfree fully electronic nucleic acid detection system based on a field-effect transistor device," Biosensors and Bioelectronics, vol. 19, no. 12, pp. 1723-1731, 2004.

[19] F. Yan, M. Zhang, and J. Li, "Solution-gated graphene transistors for chemical and biological sensors," Advanced Healthcare Materials, 2013.
[20] H. Berney, J. West, E. Haefele, J. Alderman, W. Lane, and J. K. Collins, "DNA diagnostic biosensor: development, characterisation and performance," Sensors and Actuators B, vol. 68, no. 1 , pp. 100-108, 2000.

[21] F. Pouthas, C. Gentil, D. Côte, and U. Bockelmann, "DNA detection on transistor arrays following mutation-specific enzymatic amplification," Applied Physics Letters, vol. 84, no. 9, pp. 15941596, 2004.

[22] L. Gu, J. Han, H. Zhang, and X. Chen, "DNA field effect transistor," in Proceedings of the International Conference on Sensor Technology (ISTC '01), pp. 47-49, International Society for Optics and Photonics, October 2001.

[23] A. Sassolas, B. D. Leca-Bouvier, and L. J. Blum, "DNA biosensors and microarrays," Chemical Reviews, vol. 108, no. 1, pp. 109139, 2008.

[24] T. G. Drummond, M. G. Hill, and J. K. Barton, "Electrochemical DNA sensors," Nature Biotechnology, vol. 21, no. 10, pp. 11921199, 2003.

[25] F. Schwierz, "Graphene transistors," Nature Nanotechnology, vol. 5, no. 7, pp. 487-496, 2010.

[26] A. K. Geim and A. H. MacDonald, "Graphene: exploring carbon flatland," Physics Today, vol. 60, no. 8, pp. 35-41, 2007.

[27] A. K. Geim and K. S. Novoselov, "The rise of graphene," Nature Materials, vol. 6, no. 3, pp. 183-191, 2007.

[28] T. Kurkina, A. Vlandas, A. Ahmad, K. Kern, and K. Balasubramanian, "Label-free detection of few copies of DNA with carbon nanotube impedance biosensors," Angewandte Chemie International Edition, vol. 50, no. 16, pp. 3710-3714, 2011.

[29] I. Heller, A. M. Janssens, J. Männik, E. D. Minot, S. G. Lemay, and C. Dekker, "Identifying the mechanism of biosensing with carbon nanotube transistors," Nano Letters, vol. 8, no. 2, pp. 591595, 2008.

[30] F. Karimi, M. Ahmadi, M. Rahmani, E. Akbari, M. J. Kiani, and M. Khalid, "Analytical modeling of graphene-based DNA sensor," Science of Advanced Materials, vol. 4, no. 11, pp. 11421147, 2012.

[31] I. Heller, S. Chatoor, J. Männik, M. A. G. Zevenbergen, C. Dekker, and S. G. Lemay, "Influence of electrolyte composition on liquid-gated carbon nanotube and graphene transistors," Journal of the American Chemical Society, vol. 132, no. 48, pp. 17149-17156, 2010.

[32] J. Klimeš and A. Michaelides, "Perspective: advances and challenges in treating van der Waals dispersion forces in density functional theory," The Journal of Chemical Physics, vol. 137, Article ID 120901, 12 pages, 2012.

[33] A. Poghossian, A. Cherstvy, S. Ingebrandt, A. Offenhäusser, and M. J. Schöning, "Possibilities and limitations of labelfree detection of DNA hybridization with field-effect-based devices," Sensors and Actuators B, vol. 111-112, pp. 470-480, 2005.

[34] H. K. F. Abadi, R. Yusof, S. M. Eshrati et al., "Current-voltage modeling of graphene-based DNA sensor," Neural Computing and Applications, 2013.

[35] M. J. Kiani, M. T. Ahmadi, H. K. F. Abadi, M. Rahmani, and A. Hashim, "Analytical modelling of monolayer graphene-based ion-sensitive FET to $\mathrm{pH}$ changes," Nanoscale Research Letters, vol. 8, no. 1, pp. 1-9, 2013.

[36] S. Datta, Electronic Transport in Mesoscopic Systems, Cambridge University Press, Cambridge, UK, 2002.

[37] D. S. L. Abergel, V. Apalkov, J. Berashevich, K. Ziegler, and T. Chakraborty, "Properties of graphene: a theoretical perspective," Advances in Physics, vol. 59, no. 4, pp. 261-482, 2010. 
[38] A. F. Ávila, A. S. Neto, and H. Nascimento Junior, "Hybrid nanocomposites for mid-range ballistic protection," International Journal of Impact Engineering, vol. 38, no. 8-9, pp. 669675, 2011.

[39] M. T. Ahmadi, Z. Johari, N. A. Amin, A. H. Fallahpour, and R. Ismail, "Graphene nanoribbon conductance model in parabolic band structure," Journal of Nanomaterials, vol. 2010, Article ID 753738, 4 pages, 2010.

[40] B. Huang, N. Tai, and W. Huang, "Optimization and coordination of HAFDV PINN control by improved PSO," Journal of Control Science and Engineering, vol. 2013, Article ID 872624, 7 pages, 2013.

[41] W. He, Y. Cheng, L. Xia, and F. Liu, "A new particle swarm optimization-based method for phase unwrapping of MRI data," Computational and Mathematical Methods in Medicine, vol. 2012, Article ID 475745, 9 pages, 2012.

[42] R. Rahmani, A. Khairuddin, S. M. Cherati, and H. A. Mahmoud Pesaran, "A novel method for optimal placing wind turbines in a wind farm using Particle Swarm Optimization (PSO)," in Proceedings of the 9th International Power and Energy Conference (IPEC '10), pp. 134-139, October 2010.

[43] R. Rahmani, M. F. Othman, R. Yusof, and M. Khalid, "Solving economic dispatch problem using particle swarm optimization by an evolutionary technique for initializing particles," Journal of Theoretical and Applied Information Technology, vol. 46, no. 2, pp. 526-536, 2012.

[44] J. Lv, Y. Wang, L. Zhu, and Y. Ma, "Particle-swarm structure prediction on clusters," The Journal of Chemical Physics, vol. 137, Article ID 084104, 2012.

[45] S. Das, A. Abraham, and A. Konar, "Particle swarm optimization and differential evolution algorithms: technical analysis, applications and hybridization perspectives," in Advances of Computational Intelligence in Industrial Systems, vol. 116 of Studies in Computational Intelligence, pp. 1-38, Springer, Berlin, Germany, 2008.

[46] D. W. Boeringer and D. H. Werner, "Particle swarm optimization versus genetic algorithms for phased array synthesis," IEEE Transactions on Antennas and Propagation, vol. 52, no. 3, pp. 771-779, 2004.

[47] M. Clerc, Particle Swarm Optimization, Wiley-ISTE, 2006.

[48] R. C. Eberhart and Y. Shi, "Particle swarm optimization: developments, applications and resources," in Proceedings of the Congress on Evolutionary Computation, pp. 81-86, May 2001.

[49] Y. Wang, J. Lv, L. Zhu, and Y. Ma, "Crystal structure prediction via particle-swarm optimization," Physical Review B, vol. 82, no. 9, Article ID 094116, 2010.

[50] R. C. Eberhart, Y. Shi, and J. Kennedy, Swarm Intelligence, Elsevier, Indianapolis, Ind, USA, 2001.

[51] R. Poli, J. Kennedy, and T. Blackwell, "Particle swarm optimization," Swarm Intelligence, vol. 1, no. 1, pp. 33-57, 2007.

[52] Y. del Valle, G. K. Venayagamoorthy, S. Mohagheghi, J.-C. Hernandez, and R. G. Harley, "Particle swarm optimization: basic concepts, variants and applications in power systems," IEEE Transactions on Evolutionary Computation, vol. 12, no. 2, pp. 171-195, 2008. 

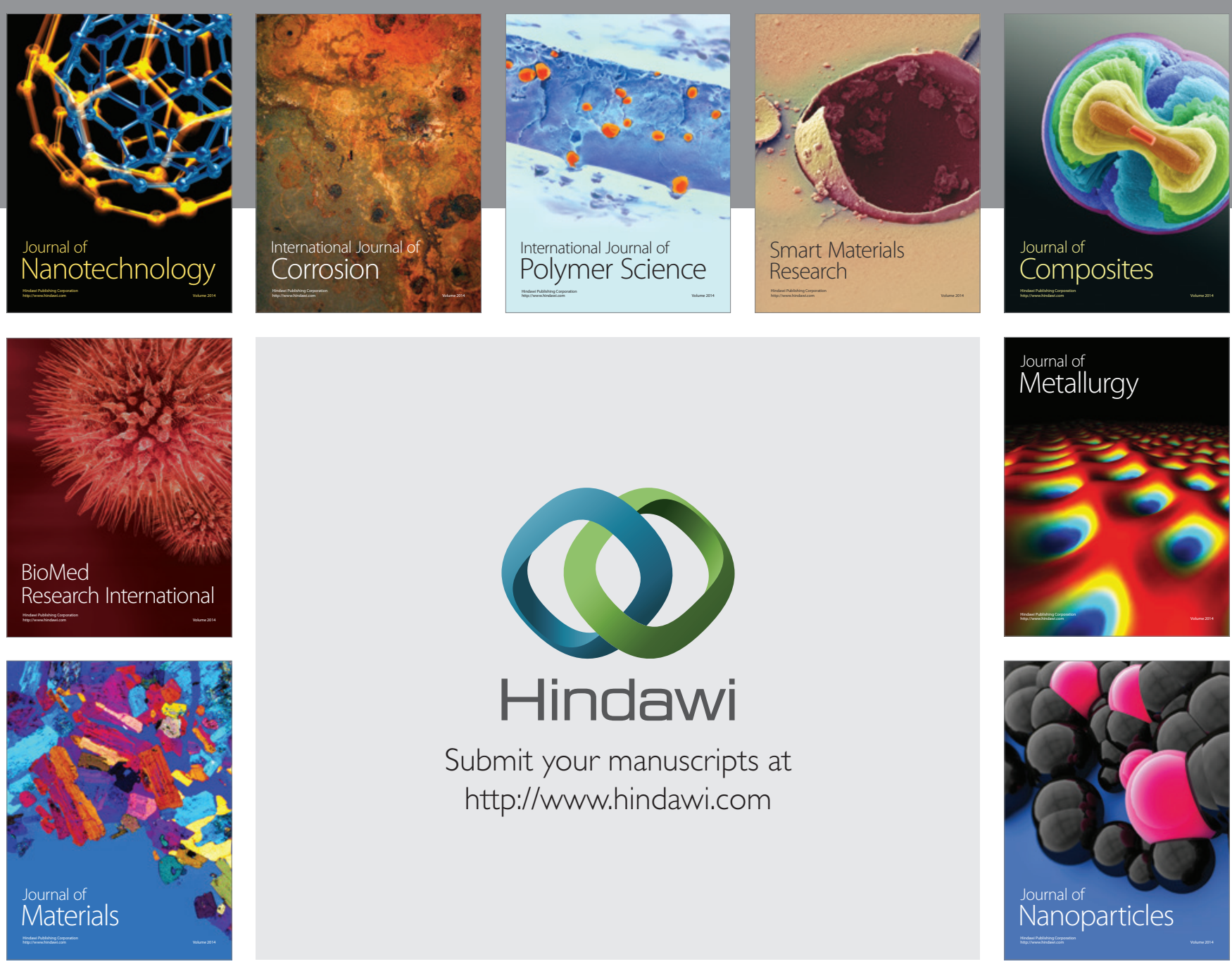

Submit your manuscripts at http://www.hindawi.com
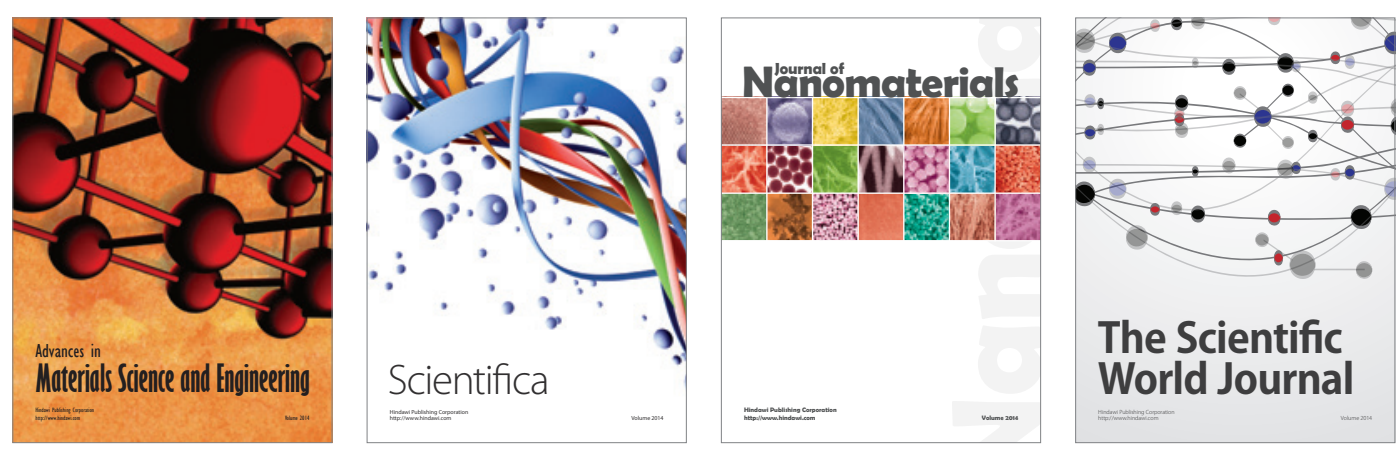

\section{The Scientific World Journal}
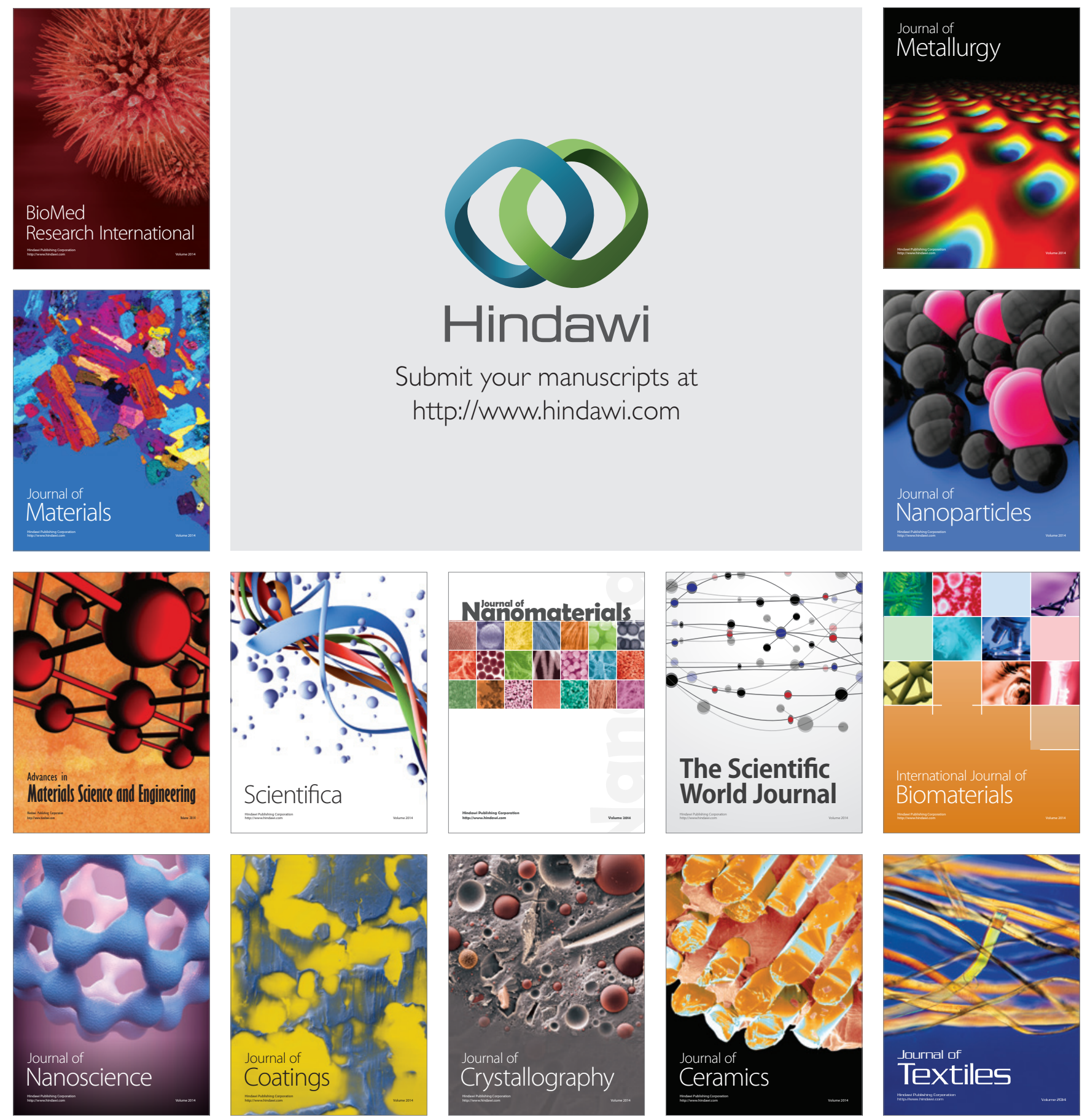